\title{
Involvement of NEK2 and its interaction with NDC80 and CEP250 in hepatocellular carcinoma
}

\author{
Lu Zeng ${ }^{1,2}$, Xiude Fan ${ }^{1}$, Xiaoyun Wang ${ }^{1}$, Huan Deng ${ }^{1}$, Xiaoge Zhang ${ }^{1}$, Kun Zhang ${ }^{1}$, Shan He ${ }^{1,2}, \mathrm{Na} \mathrm{Li}^{1}$, \\ Qunying Han ${ }^{1}$ and Zhengwen Liu ${ }^{1 *}$ (D)
}

\begin{abstract}
Background: NEK2 has an established involvement in hepatocellular carcinoma (HCC) but the roles of NEK2 and its interacting proteins in HCC have not been systematically explored.

Methods: This study examined NEK2 and its interacting proteins in HCC based on multiple databases.

Results: NEK2 mRNA was highly expressed in HCC tissues compared with normal liver tissues. The survival of HCC patients with high NEK2 mRNA expression was shorter than those with low expression. MAD1L1, CEP250, MAPK1, NDC80, PPP1CA, PPP1R2 and NEK11 were the interacting proteins of NEK2. Among them, NDC80 and CEP250 were the key interacting proteins of NEK2. Mitotic prometaphase may be the key pathway that NEK2 and its interacting proteins contributed to HCC pathogenesis. NEK2, NDC80 and CEP250 mRNAs were highly expressed in HCC tissues compared with normal liver tissues. The mRNA levels of NEK2 were positively correlated with those of NDC80 or CEP250. Univariate regression showed that NEK2, NDC80 and CEP250 mRNA expressions were significantly associated with HCC patients' survival. Multivariate regression showed that NDC80 mRNA expression was an independent predictor for HCC patients' survival. Methylations and genetic alterations of NEK2, NDC80 and CEP250 were observed in HCC samples. The alterations of NEK2, NDC80 and CEP250 genes were co-occurrence. Patients with high mRNA expression and genetic alterations of NEK2, NDC80 and CEP250 had poor prognosis.
\end{abstract}

Conclusions: NEK2 and its interacting proteins NDC80 and CEP250 play important roles in HCC development and progression and thus may be potentially used as biomarkers and therapeutic targets of HCC.

Keywords: NEK2, NDC80, CEP250, Hepatocellular carcinoma, Biomarker

\section{Background}

Hepatocellular carcinoma (HCC) has high morbidity and mortality and is the third leading cause of cancerassociated deaths worldwide [1]. Characteristics of HCC include aggressive, high malignancy, early metastasis and poor prognosis. With the application of alpha-fetoprotein

\footnotetext{
*Correspondence: liuzhengwen113@xjtu.edu.cn

${ }^{1}$ Department of Infectious Diseases, First Affiliated Hospital of Xi'an Jiaotong University, No. 277 Yanta West Road, Xi'an 710061, Shaanxi Province, People's Republic of China

Full list of author information is available at the end of the article
}

(AFP) and improvement of imaging techniques such as ultrasound and computerized tomography and the progress of surgical and interventional therapy for HCC, the early diagnostic rate of $\mathrm{HCC}$ and the prognosis of $\mathrm{HCC}$ patients appear to have been increased and improved. However, many patients were diagnosed at the advanced stage of $\mathrm{HCC}$ and had poor prognosis. The 5-year survival rate in patients with early stage HCC is more than $70 \%$, but it is less than $5 \%$ in patients with advanced $\mathrm{HCC}$ [2]. Therefore, the exploration of biomarkers for the early 
diagnosis and targets for the therapy of $\mathrm{HCC}$ is an important research focus.

Never in mitosis gene A-related kinase 2 (NEK2) belongs to serine/threonine kinase and widely exists in the centrosome of cells [3]. NEK2 plays important roles in regulating the mitosis process, including chromatin separation, spindle assembly, centrosome division and tumor drug resistance [4-6]. Studies have shown that the high mRNA expression of NEK2 was related to the incidence, differentiation, metastasis and prognosis of multiple myeloma [7], pancreatic ductal adenocarcinoma [8], ovarian cancer [9], colorectal cancer [10] and breast cancer [11]. Studies also showed the involvement of NEK2 in the development and progression of HCC [12] and overexpression of $N E K 2$ was indicated to be associated with a poor survival in HCC patients [13]. However, most of the previous studies were conducted in small sample sizes of patients and the possible network proteins of NEK2 remain to be investigated. Therefore, this study systematically examined the role of NEK2 in HCC, mined the NEK2 interacting proteins and explored the underlying mechanisms by the analyses of multiple databases to provide evidence for the potential of NEK2 and its interacting proteins in the diagnosis and therapy of HCC.

\section{Methods}

Multiple databases were used to systematically investigate the potential involvement of NEK2 and its interacting proteins in HCC and explored the possible mechanisms. First, the mRNA expression of NEK2 in HCC and the association of NEK2 mRNA expression levels with prognosis of HCC patients were analyzed by UALCAN. Second, the upstream or downstream interacting proteins of NEK2 were mined using GeneSense and the relationships between NEK2 and its interacting proteins and the key interacting proteins were verified and identified by STRING (Search Tool for the Retrieval of Interacting Genes/Proteins). Third, the functional enrichment pathways of NEK2 and its interacting proteins were explored by DAVID (the Database for Annotation, Visualization and Integrated Discovery) and KOBAS (KEGG Orthology Based Annotation System) and the pathways of NEK2 and its interacting proteins were verified using Reactome. Fourth, the effects of NEK2 and its key interacting proteins on HCC patients' survival were examined using data downloaded from TCGA (The Cancer Genome Atlas) and the mRNA expression of NEK2, NDC80 and CEP250 in HCC were analyzed by Oncomine. Lastly, the genetic alterations of $N E K 2$ and the genes of NEK2 key interacting proteins in $\mathrm{HCC}$, the relationship of the genetic alterations between the genes, and the influence of the genetic alterations on HCC patients' survival were analyzed by cBioPortal. The flow chart of the study was shown in Additional file 1: Figure S1.

\section{UALCAN database}

UALCAN (https://ualcan.path.uab.edu/analysis.html) is a user-friendly and interactive website, which provides easy access to publicly available cancer transcriptome data (TCGA and MET500 transcriptome sequencing). Plots depicting gene expression and patient survival information based on different gene expression can be obtained by UALCAN. This study explored the mRNA expression of NEK2 in HCC in comparison with normal liver, and plotted the survival curve of $\mathrm{HCC}$ patients with different mRNA expression levels of NEK2 by UALCAN.

\section{GeneSense database}

GeneSense (https://www.biomedsense.org/genesense. php) is a web-based platform that allows users to construct, visualize, manipulate and analyze gene information. It aims to assist researchers to find the optimal gene regulatory factors by associated networks. GeneSense adopts a number of methods to annotate protein-protein interaction (PPI). The PPI network which GeneSense can construct has three styles, namely nodenet, leafnet and loopnet. Nodenet supports basic queries of PPI network for upstream or downstream protein analysis. Leafnet model visualizes the complexity of the queried protein and its upstream or downstream proteins. Loopnet visualizes the upstream and downstream target of the queried protein. This study used loopnet style to visualize the possible upstream and downstream interacting proteins of NEK2 and both the upstream and downstream proteins were all considered to be NEK2 interacting proteins.

STRING: Protein-protein interaction network construction STRING: functional protein association networks (https ://string-db.org/), is a website which can predict PPIs. We used STRING to verify PPI network of NEK2 and its interacting proteins obtained from GeneSense. An interaction score $>0.9$ was regarded as high confidence PPIs and the related interacting protein was considered to be a key interacting protein of NEK2.

\section{DAVID and KOBAS functional enrichment analyses}

The functional and signaling pathway analyses of NEK2 and its interacting proteins were performed on two public database platforms, DAVID (https://david.ncifc rf.gov/), and KOBAS 3.0 (https://kobas.cbi.pku.edu.cn/ index.php). DAVID database was used to acquire the Gene Ontology (GO) terms that include three categories, namely molecular function (MF), cellular component (CC) and biological process (BP). KOBAS 3.0 database was used to obtain the main Kyoto Encyclopedia of 
Genes and Genomes (KEGG) pathways. The enriched GO terms and KEGG pathways of NEK2 and its interacting proteins were visualized by $\mathrm{R}$ software and Cytoscape 3.6.1.

\section{Reactome database}

Reactome database (https://reactome.org/what-is-react ome) provides intuitive bioinformatics tools for the visualization, interpretation and analysis of pathway knowledge, and can present data which describe possible pathways if annotated proteins and small molecules were present in a cell. The possible pathways that interesting proteins can be obtained and the details of the pathways can be visualized. This study used the ReactomeFIPlugIn in Cytoscape software to analyze the pathways that NEK2 and its interacting proteins may involve in and to acquire the pathway diagram.

\section{TCGA database}

The Cancer Genome Atlas (TCGA) database (https:// cancergenome.nih.gov/), which integrates gene expression data and clinical data, was used to verify and analyze the prognostic value of NEK2 and its interacting proteins in HCC patients. R "survival" package [14] was used to visualize the results. The clinical covariates of 373 HCC patients from TCGA database were included in Cox proportional hazard model to identify the independent predictor for HCC patients' survival. The correlations of mRNA expression between $N E K 2$ and NDC80 or CEP250 were analyzed.

\section{Oncomine database}

Oncomine database (https://www.oncomine.org/resou rce/login.html), an online microarray database, was used to explore and confirm the mRNA expression levels of $N E K 2$ and the genes of NEK2 key interacting proteins in HCC. The conditions for filter setting were as follows: Gene: "NEK2 or its key interacting proteins", Analysis Type: "Cancer vs. Normal Analysis", Cancer Type: "Hepatocellular Carcinoma", Data Type: "mRNA", Sample Type: "Clinical Specimen". The studies incorporating mRNA expression data of NEK2 and the genes of NEK2 key interacting proteins were obtained, and ordered by "Over-expression: Gene Rank". The different studies could be compared by conducting meta-analysis to demonstrate the reliability of these data.

\section{CBioPortal database}

cBioPortal database (https://www.cbioportal.org/) provides visualization tools for research and analysis of cancer gene data. The integrated genomic data types include somatic cell mutation, DNA copy number changes, mRNA and microRNA expression, DNA methylation, protein abundance and phosphoprotein abundance. The genetic alterations, relationship between genetic alterations, methylation, co-expression and the survival of HCC patients in relation to NEK2 and its key interacting proteins were explored through the database. "Liver Hepatocellular Carcinoma (TCGA, Provisional)" study was selected for analyzing, and there were 366 patients/ samples with mutation and copy number alteration (CNA) data which comprise "mutation, putative copynumber alterations" from GISTIC, and mRNA expression z-Scores (RNA Seq V2 RSEM)" information. The mRNA expression z-Score threshold was \pm 2.0 .

\section{Statistical analysis}

Statistical analyses were carried out by SPSS statistical software (Version 22.0; Inc., IBM, NY, USA) and Graghpad Prism software (Version 6.0; Inc., Graghpad, San Diego, CA, USA). The mRNA expression was expressed as mean \pm standard deviation (SD), and the expression differences between groups were compared by nonparametric test. Cox regression model was used to identify independent predictors for HCC patients' survival. Survival of HCC patients was estimated using the KaplanMeier method and compared by the log-rank test. The mRNA expression correlation between NEK2 and other genes were evaluated by Spearman correlation analyses. A $p$ value of $<0.05$ was considered statistically significant.

\section{Results \\ mRNA expression of NEK2 in normal and HCC tissues and its effect on HCC patient survival by UALCAN}

Based on UALCAN analyses [15], the mRNA expression of NEK2 was elevated in HCC tissues $(\mathrm{n}=371)$ compared with normal liver tissues $\left(\mathrm{n}=50, p=1.625 \times 10^{-12}\right.$, Fig. 1a). Survival analysis of patients with different levels of NEK2 mRNA expression demonstrated that the survival time of patients with high $N E K 2$ mRNA expression $(\mathrm{n}=89)$ was shorter than those with low expression $(\mathrm{n}=276, p=0.012$, Fig. $1 \mathrm{~b})$.

\section{The upstream and downstream interacting proteins of NEK2 by Genesense}

The upstream and downstream interacting proteins of NEK2 were mined by Genesense website [16]. MAD1L1 [MAD1 mitotic arrest deficient-like 1 (yeast)] [17] was found to be the upstream protein of NEK2 while CEP250 (centrosomal protein $250 \mathrm{kDa}$ ) [18], MAPK1 (mitogenactivated protein kinase 1) [19], NDC80 (nuclear division cycle 80 ) [20], PPP1R2 [protein phosphatase 1, regulatory (inhibitor) subunit 2] [21] and PPP1CA (protein phosphatase 1 catalytic subunit alpha) were the downstream proteins of NEK2. NEK11 [NIMA (never in mitosis gene 

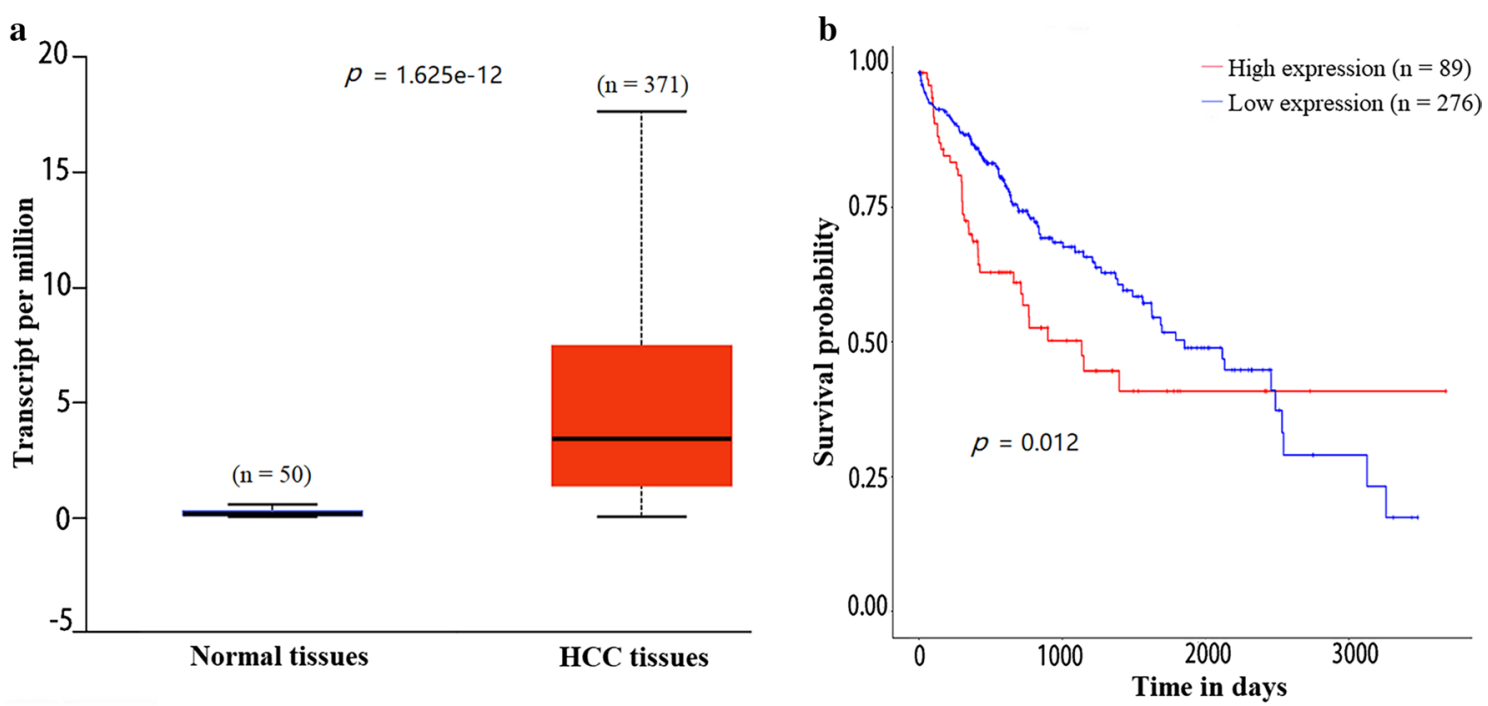

Fig. 1 The mRNA expression of NEK2 in normal tissues and HCC tissues (a) and the effect of NEK2 mRNA expression on HCC patients' survival (b) by UALCAN analysis

a)- related kinase 11] [22] was indicated to be both the upstream and downstream protein of NEK2 (Fig. 2a).

\section{Protein-protein interaction by STRING database}

The PPI network was constructed by STRING database [23] to verify the association between NEK2 and its interacting proteins. The results showed that NEK2 had strong interaction with its downstream and upstream proteins. The PPI network's enrichment $p$-value was $1.23 \mathrm{e}-07$. Among these proteins, NDC80 [24, 25] and CEP250 [26] had the highest interaction scores (Fig. 2b). The interaction scores between NEK2 and NDC80, and NEK2 and CEP250 were 0.994 and 0.995 , respectively, indicating that NDC80 and CEP250 were NEK2 key interacting proteins. The main GO and KEGG pathways involving NEK2 and its interacting proteins analyzed by STRING database were downloaded (Table 1). Cell cycle [false discovery rate $(F D R)=2.78 \mathrm{E}-05]$, regulation of mitotic cell cycle phase transition $(\mathrm{FDR}=4.01 \mathrm{E}-05)$ and mitotic cell cycle process $(F D R=0.0002)$ were the main GO biological progress pathways. Phosphatase binding $\quad(F D R=0.0026)$, protein serine/threonine
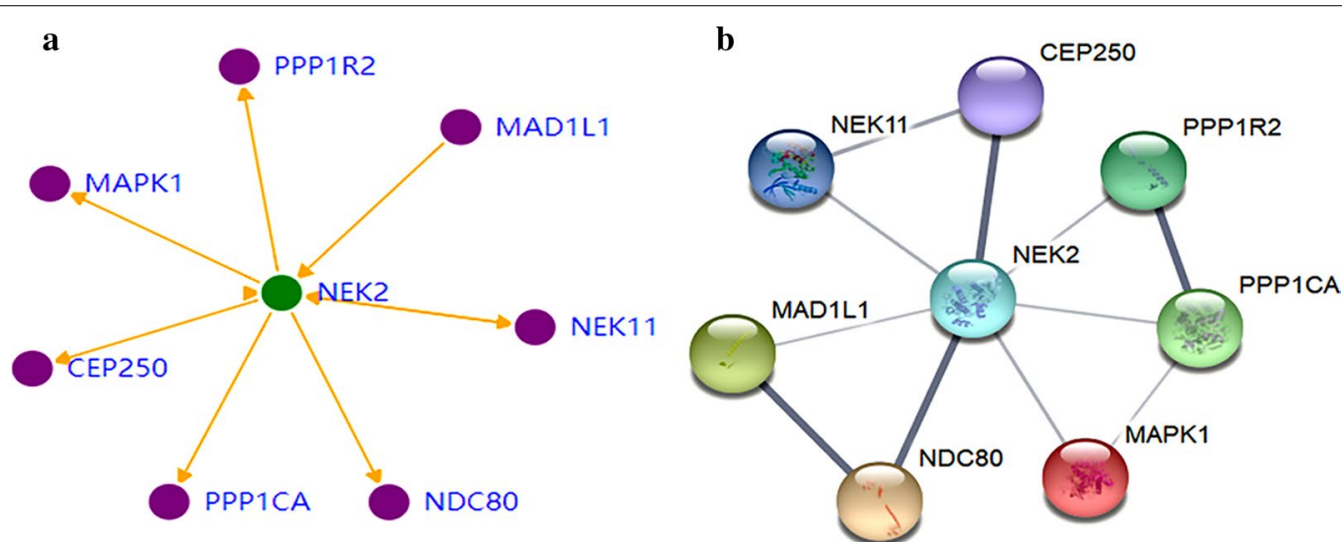

Fig. 2 The upstream and downstream interacting proteins of NEK2 by Genesense analysis and the protein-protein interaction network of NEK2 and its interacting proteins by STRING database. a The upstream and downstream interacting proteins of NEK2 by Genesense analysis. The protein with an arrow pointing to NEK2 is the upstream protein of NEK2, and the protein with an arrow pointing away from NEK2 is the downstream protein of NEK2. $\mathbf{b}$ The protein-protein interaction network of NEK2 and its interacting proteins by STRING database. The line between proteins indicates that proteins interact with each other, and the thickness of the line indicates the interaction score 
Table 1 The main GO and KEGG pathways involving NEK2 and its interacting proteins downloaded from STRING database

\begin{tabular}{|c|c|c|c|c|c|}
\hline Categories & Pathway ID & Pathway description & Gene count & FDR & Genes \\
\hline \multirow[t]{3}{*}{ Biological process (GO) } & GO:0007049 & Cell cycle & 7 & $2.78 \mathrm{E}-05$ & $\begin{array}{l}\text { CEP250,MAD1L1,MAPK1,NDC80,NEK1 } \\
\text { 1,NEK2,PPP1CA }\end{array}$ \\
\hline & GO:1901990 & Regulation of mitotic cell cycle phase transition & 5 & 4.01E-05 & CEP250,MAD1L1,NDC80,NEK11,NEK2 \\
\hline & GO:1903047 & Mitotic cell cycle process & 5 & 0.00021 & CEP250,MAD1L1,NDC80,NEK11,NEK2 \\
\hline \multirow[t]{3}{*}{ Molecular function (GO) } & GO:0019902 & Phosphatase binding & 3 & 0.0026 & MAPK1,NEK2,PPP1CA \\
\hline & GO:0004674 & Protein serine/threonine kinase activity & 3 & 0.0219 & MAPK1,NEK11,NEK2 \\
\hline & GO:0019903 & Protein phosphatase binding & 2 & 0.0265 & $N E K 2, P P P 1 C A$ \\
\hline \multirow[t]{3}{*}{ Cellular component (GO) } & GO:0005815 & Microtubule organizing center & 5 & 0.00037 & CEP250,MAD1L1,MAPK1,NDC80,NEK2 \\
\hline & GO:0005813 & Centrosome & 4 & 0.00059 & CEP250,MAD1L1,NDC80,NEK2 \\
\hline & GO:0000777 & Condensed chromosome kinetochore & 3 & 0.00059 & MAD1L1,NDC80,NEK2 \\
\hline \multirow[t]{3}{*}{ KEGG pathways } & hsa04720 & Long-term potentiation & 2 & 0.0337 & MAPK1,PPP1CA \\
\hline & hsa04914 & Progesterone-mediated oocyte maturation & 2 & 0.0356 & MAD1L1,MAPK1 \\
\hline & hsa04022 & cGMP-PKG signaling pathway & 2 & 0.0358 & MAPK1,PPP1CA \\
\hline
\end{tabular}

FDR false discovery rate

kinase activity $(\mathrm{FDR}=0.0219)$ and protein phosphatase binding $(F D R=0.0265)$ were the main GO molecular function pathways. Microtubule organizing center $(F D R=0.00037)$, centrosome $(F D R=0.00059)$ and condensed chromosome kinetochore $(F D R=0.00059)$ were the main GO cellular component pathways. Long-term potentiation $\quad(\mathrm{FDR}=0.0337)$, progesterone-mediated oocyte maturation $(\mathrm{FDR}=0.0356)$ and cGMP-PKG signaling pathway $(\mathrm{FDR}=0.0358)$ were the main $\mathrm{KEGG}$ pathways. The proteins enriched in each pathways were displayed in Table 1.

Functional enrichment by DAVID and KOBAS 3.0 databases In order to further analyze the functional enrichment of NEK2 and its interacting proteins (MAD1L1, CEP250, MAPK1, NDC80, NEK11, PPP1CA and PPP1R2) in HCC, GO and KEGG pathways were investigated based on
DAVID [27] and KOBAS 3.0 [28] databases. The details of the main GO terms and KEGG pathways were shown in Table 2, and the main GO terms and KEGG pathways were demonstrated in Fig. 3a and Fig. 3b, respectively. Phosphorus metabolic process $(p=0.001496693)$, phosphate metabolic process $(p=0.001496693)$, and cell cycle $(p=0.005071148)$ were significantly enriched in the category of GO biological progress. Protein serine/threonine kinase activity $(p=0.005262684)$ was the mainly enriched pathway in the category of GO molecular function. There were no pathways enriched in GO cellular component. Long-term potentiation $(p=0.000150473)$, oocyte meiosis $(p=0.000484062)$, vascular smooth muscle contraction $(p=0.000508059)$, platelet activation $(p=0.000524374)$ and insulin signaling pathway ( $p=0.000663998)$ were found to be the significantly enriched KEGG pathways (Table 2). MAPK1 and

Table 2 The main GO and KEGG pathways associated with NEK2 and its interacting proteins by DAVID and KOBAS 3.0 databases analyses

\begin{tabular}{llllll}
\hline Categories & Pathway ID & Pathway description & Gene count & $\boldsymbol{p}$-value & Genes \\
\hline Biological process (GO) & G0:0006793 & Phosphorus metabolic process & 4 & 0.0015 & MAPK1, PPP1CA, NEK2, NEK11 \\
& G0:0006796 & Phosphate metabolic process & 4 & 0.0015 & MAPK1, PPP1CA, NEK2, NEK11 \\
& GO:0007049 & Cell Cycle & 3 & 0.00507 & MAPK1, PPP1CA, NEK2 \\
Molecular function (GO) & GO:0004674 & Protein serine/threonine kinase activity & 3 & 0.00526 & MAPK1, NEK2, NEK11 \\
KEGG pathways & hsa04720 & Long-term potentiation & 2 & 0.00015 & MAPK1, PPP1CA \\
& hsa04114 & Oocyte meiosis & 2 & 0.00048 & MAPK1, PPP1CA \\
& hsa04270 & Vascular smooth muscle contraction & 2 & 0.00051 & MAPK1, PPP1CA \\
& hsa04611 & Platelet activation & 2 & 0.00052 & MAPK1, PPP1CA \\
& hsa04910 & Insulin signaling pathway & 2 & 0.00066 & MAPK1, PPP1CA \\
\hline
\end{tabular}



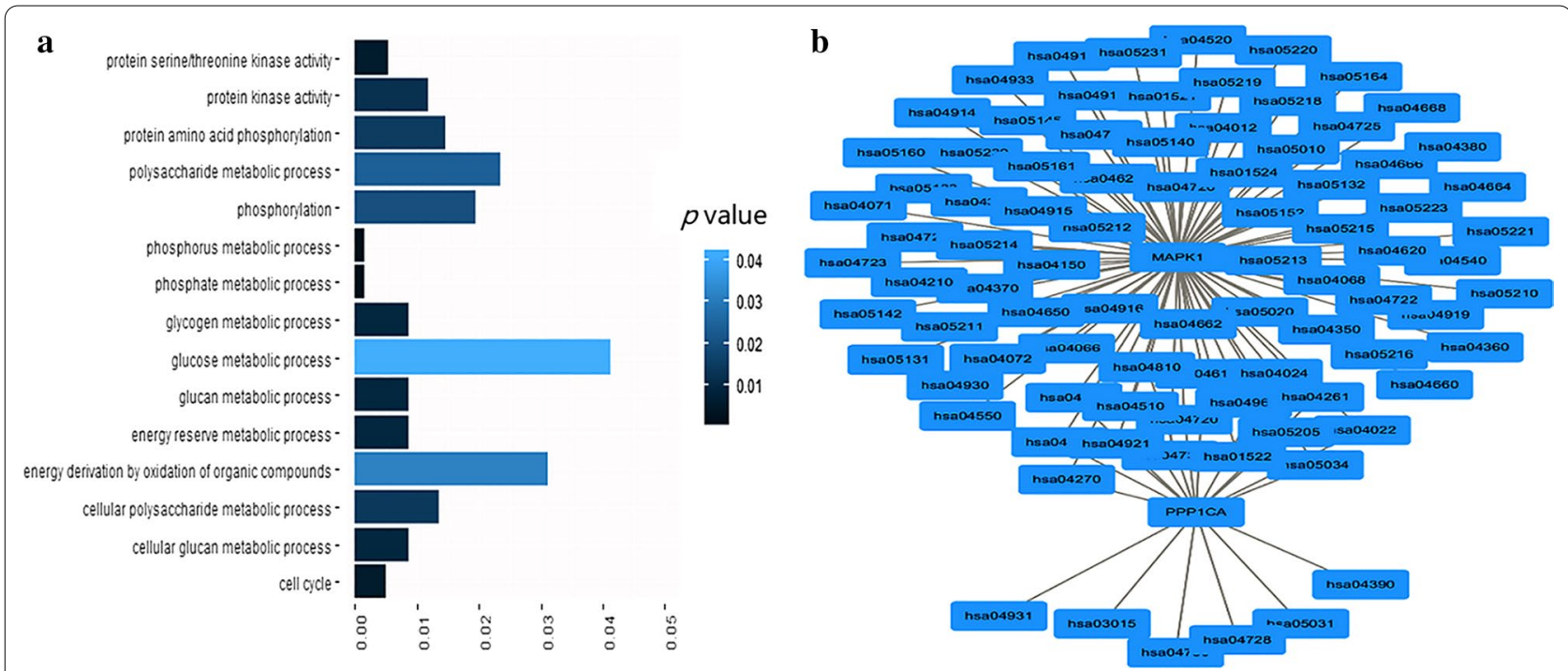

Fig. 3 The main GO terms that NEK2 and its interacting proteins enriched and the main KEGG pathways that NEK2 and its interacting proteins enriched. a The main GO terms that NEK2 and its interacting proteins enriched. The shorter the bar graph and the darker the color of each term, the greater the statistical difference is. $\mathbf{b}$ The main KEGG pathways that NEK2 and its interacting proteins enriched. MAPK1 and PPP1CA enriched the most KEGG pathways

PPP1CA were the major proteins involved in the KEGG pathways (Fig. 3b).

\section{Reactome analyses}

Reactome dababase [29] was used to verify the pathways that NEK2 and its interacting proteins were likely to participate in. The Analyze Data tool of Reactome was used to perform the analyses. NEK2 and its interacting proteins list were inputs. Human was chosen as species. Results showed that there were 122 significant pathways which NEK2 and its interacting proteins involved in $(p<0.05)$ (Additional file 2: Figure S2). The top 5 pathways were displayed in Table 3. Among the results, mitotic prometaphase $(p=2.63 \mathrm{E}-06), \mathrm{M}$ phase $(p=2.24 \mathrm{E}-05)$, and cell cycle, mitotic $(p=9.11 \mathrm{E}-05)$ were the three pathways including the most queried genes. The details of mitotic prometaphase pathway were shown in Additional file 3: Figure S3. The main biological processes that NEK 2 and its interacting proteins involved in included recruitment of NuMA (nuclear mitotic apparatus protein) to mitotic centrosomes, kinetochore capture of astral microtubules, resolution of sister chromatid cohesion and condensation of prometaphase chromosomes.

\section{Survival analyses by TCGA database}

Clinical data of HCC patients were downloaded from TCGA database [30] and the effects of different mRNA expression levels of NEK2 and the genes of NEK2 interacting proteins on prognosis of HCC patients were investigated. A total of $373 \mathrm{HCC}$ patients' clinical data from TCGA database were downloaded, and the patients were divided into high mRNA expression group $(n=187)$ and low mRNA expression group $(n=186)$ based on the median mRNA expression value of interesting genes. Only NEK2, NDC80 and CEP250 were found to be associated with the survival of HCC patients $\left(P=6 \times 10^{-5}\right.$, $P=0.00122$ and $P=0.00073$, respectively, Fig. 4). The

Table 3 The top 5 pathways associated with NEK2 and its interacting proteins by Reactome analyses

\begin{tabular}{|c|c|c|c|c|}
\hline Pathway description & Gene count & $p$ value & FDR & Genes \\
\hline Mitotic prometaphase & 4 & $2.63 \mathrm{E}-06$ & $3.52 \mathrm{E}-04$ & CEP250,NEK2,MAD1L1,NDC80 \\
\hline M phase & 4 & $2.24 \mathrm{E}-05$ & $1.50 E-03$ & CEP250,NEK2,MAD1L1,NDC80 \\
\hline Cell cycle, mitotic & 4 & $9.11 \mathrm{E}-05$ & 4.01E-03 & CEP250,NEK2,MAD1L1,NDC80 \\
\hline RHO GTPase effectors & 3 & $3.20 E-04$ & 0.0106 & MAPK1,MAD1L1,NDC80 \\
\hline Opioid signaling & 2 & $7.85 \mathrm{E}--04$ & 0.0123 & MAPK1,PPP1CA \\
\hline
\end{tabular}

FDR false discovery rate 

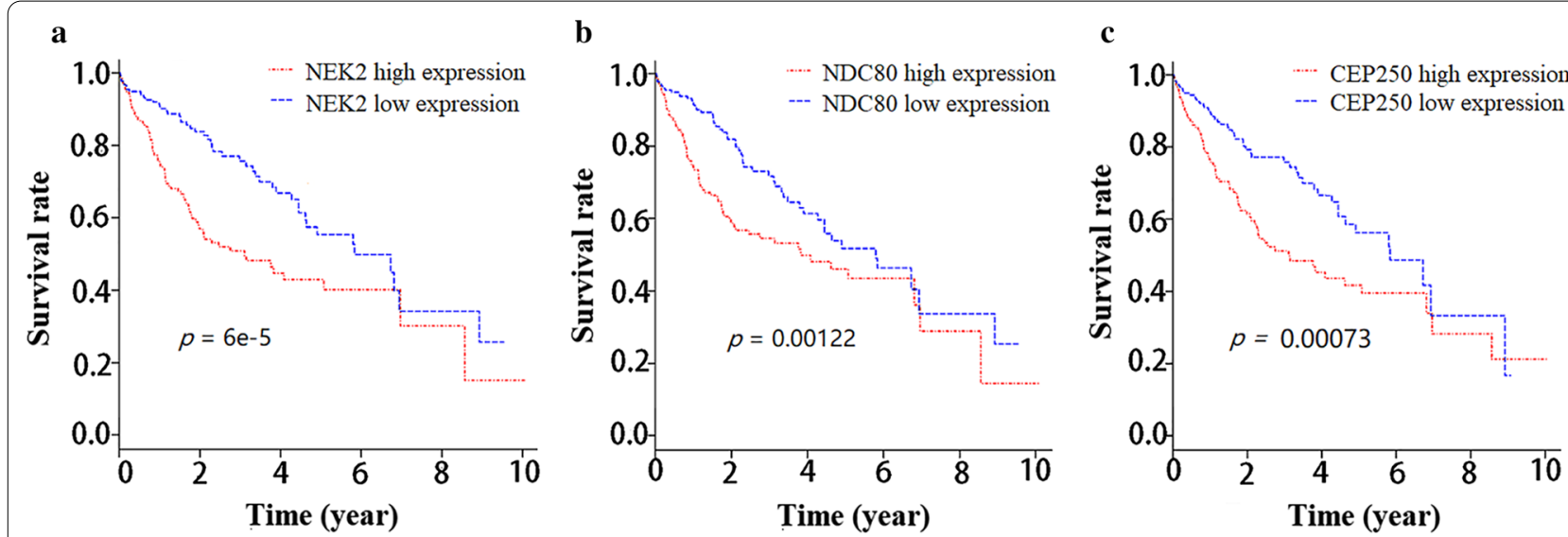

Fig. 4 Effect of different NEK2 (a), NDC80 (b) and CEP250 (c) mRNA expression levels on HCC patients'survival by TCGA database

detailed information of $373 \mathrm{HCC}$ patients from TCGA database were displayed in Additional file 4: Table S1. The NEK2, NDC80 and CEP250 mRNA expressions were different in relation to age, weight, recurrence status, histological grade, family HCC history, pathologic stage, tumor size, and survival time. Cox models incorporating clinical covariates of the $373 \mathrm{HCC}$ patients from TCGA database were set up. Clinical covariates and mRNA expression values of the three genes (NEK2, $N D C 80, C E P 250)$ were fitted into Cox regression models (Table 4). In univariate Cox regression analysis, HCC recurrence, pathologic stage, tumor size, and NEK2, NDC80 and CEP250 mRNA expression were all significantly associated with HCC patients' survival. In multivariate Cox regression models (the covariates with $p<0.2$ in univariate analysis were included), age, pathologic stage, and NDC80 mRNA expression were independent predictors for HCC patients' survival. The correlation between NDC80, CEP250 and NEK2 mRNA expression were analyzed. Reactome analysis revealed that CCNB1, CCNB2, and CDK1 were involved in mitotic prometaphase pathway (Additional file 3: Figure S3). Therefore, $C C N B 1, C C N B 2$, and $C D K 1$ were also included in correlation analysis. The mRNA expressions of all the five genes (NDC80, CEP250, CCNB1, CCNB2 and CDK1) were positively correlated with $N E K 2$ mRNA expression (Additional file 5: Figure S4).

\section{The mRNA expression of NEK2 and the genes of NEK2 key interacting proteins (NDC80 and CEP250) by Oncomine database}

The mRNA expression of NEK2 and the genes of NEK2 key interacting proteins (NDC80 and CEP250) in HCC was analyzed by Oncomine database [31]. There were five eligible HCC studies: Chen Liver [32], Mas Liver [33], Roessler Liver [34], Roessler Liver 2 [34] and Wurmbach
Liver [35]. The detailed information was Chen Liver: 99 HCC samples and 77 normal samples, Mas Liver: 38 HCC samples and 19 normal liver samples, Roessler Liver: $22 \mathrm{HCC}$ samples and 21 normal samples, Roessler Liver 2: 225 HCC samples and 220 normal samples, and Wurmbach Liver: 35 HCC samples and 10 normal samples. Meta-analysis of the mRNA expression level of the three genes in the five studies showed that NEK2 $\left(p=2.95 \times 10^{-67}\right), N D C 80\left(p=8.31 \times 10^{-57}\right)$ and CEP250 $(p=0.003)$ were highly expressed in HCC (Fig. 5). NEK2 was obviously over-expression in 4 studies, especially in Wurmbach Liver study. NDC80 was also obviously overexpression in 4 studies, especially in Roessler Liver and Wurmbach Liver studies. CEP250 was only obviously over-expression in Chen Liver study. None of the the three genes showed low mRNA expression in the studies.

\section{Genetic alteration and mutual exclusivity of NEK2, NDC80} and CEP250, and the effects on the survival of HCC patients by cBioPortal database

Oncoprint feature of the cBioPortal database [36] was used to determine the genetic alteration frequency and epigenetic alteration level of NEK2, NDC80 and CEP250 in HCC. The queried genes (NEK2, NDC80, CEP250) were altered in $111 / 366(30 \%)$ of queried samples. The genetic alteration frequencies of NEK2, NDC80 and CEP250 were $19 \%, 7 \%$ and $14 \%$, respectively. Among these, NEK2 has the highest frequencies of gene mutation and copy-number alterations. The detailed genetic alteration types included amplification, missense mutation, truncating mutation and mRNA high, although missense mutation and truncating mutation remained unknown significance (Fig. 6a). For epigenetic alterations, the methylation level of NEK2 and CEP250 were particularly significant in these samples (Fig. 6b). The mutual exclusivity function of cBioPortal database was used to 
Table 4 Cox regression model of clinical covariates and the NEK2, NDC80 and CEP250 mRNA expression for HCC patients' survival

\begin{tabular}{|c|c|c|c|c|c|c|}
\hline & \multicolumn{3}{|c|}{ Univariate analysis } & \multicolumn{3}{|c|}{ Multivariate analysis } \\
\hline & HR & $95 \% \mathrm{Cl}$ & $p$ value & HR & $95 \% \mathrm{Cl}$ & $p$ value \\
\hline Age & 1.012 & $0.998-1.026$ & 0.091 & 1.016 & $1.000-1.032$ & 0.045 \\
\hline Gender (ref. Female) & 1.260 & $0.884-1.795$ & 0.201 & & & \\
\hline Family history (ref. No) & 0.828 & $0.574-1.195$ & 0.314 & & & \\
\hline Recurrence (ref. No) & 0.587 & $0.371-0.926$ & 0.022 & & & \\
\hline Weight & 0.993 & $0.984-1.003$ & 0.195 & & & \\
\hline Platelet count & 1.000 & $1.000-1.000$ & 0.696 & & & \\
\hline \multicolumn{7}{|l|}{ Child-Pugh score (ref. A) } \\
\hline B & 0.469 & $0.064-3.408$ & 0.454 & & & \\
\hline C & 0.747 & $0.092-6.055$ & 0.785 & & & \\
\hline \multicolumn{7}{|l|}{ Histological grade (ref.G1) } \\
\hline G2 & 0.595 & $0.220-1.609$ & 0.306 & & & \\
\hline G3 & 0.692 & $0.277-1.731$ & 0.431 & & & \\
\hline G4 & 0.705 & $0.278-1.786$ & 0.461 & & & \\
\hline \multicolumn{7}{|l|}{ Pathologic stage (ref.Stage I) } \\
\hline Stage II & 0.178 & $0.055-0.577$ & 0.004 & 1.240 & $0.735-2.093$ & 0.420 \\
\hline Stage III & 0.254 & $0.077-0.842$ & 0.025 & 2.438 & $1.562-3.805$ & 2.438 \\
\hline Stage IV & 0.492 & $0.152-1.590$ & 0.236 & 7.033 & $2.152-22.979$ & 0.001 \\
\hline \multicolumn{7}{|l|}{ Primary tumor range (ref.T1) } \\
\hline $\mathrm{T} 2$ & 0.186 & $0.093-0.372$ & $<0.001$ & & & \\
\hline T3 & 0.267 & $0.129-0.550$ & $<0.001$ & & & \\
\hline $\mathrm{T} 4$ & 0.497 & $0.248-0.997$ & 0.049 & & & \\
\hline NEK2 mRNA expression & 1.001 & $1.000-1.001$ & 0.001 & & & \\
\hline NDC80 mRNA expression & 1.001 & $1.001-1.002$ & $<0.001$ & 1.001 & $1.001-1.002$ & $<0.001$ \\
\hline CEP250 mRNA expression & 1.000 & $1.000-1.001$ & 0.027 & & & \\
\hline
\end{tabular}

$H R$ hazard ratio, $\mathrm{Cl}$ confidence interval, Ref reference

\begin{tabular}{|c|c|c|c|c|c|c|c|}
\hline \multirow{2}{*}{\multicolumn{3}{|c|}{$\begin{array}{ccc}\text { Median Rank } & \boldsymbol{p} \text { value } & \text { Gene } \\
148.0 & 2.95 \mathrm{E}-67 & \text { NEK } 2\end{array}$}} & & & & \multirow[b]{3}{*}{4} & \multirow[b]{3}{*}{5} \\
\hline & & & & & & & \\
\hline & 1 & 2 & 3 & & \\
\hline \multicolumn{8}{|c|}{ Median Rank $\boldsymbol{p}$ value Gene } \\
\hline \multirow{2}{*}{\multicolumn{3}{|c|}{$316.0 \quad 8.31 E-57 \quad N D C 80$}} & & & & & \\
\hline & & & 1 & 2 & 3 & 4 & 5 \\
\hline \multicolumn{8}{|c|}{ Median Rank $\boldsymbol{p}$ value Gene } \\
\hline \multirow{2}{*}{5181.0} & 0.003 & CEP250 & & & & & \\
\hline & & & 1 & 2 & 3 & 4 & 5 \\
\hline \multicolumn{8}{|c|}{ 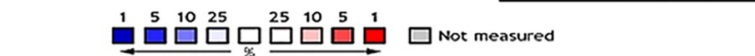 } \\
\hline \multicolumn{8}{|c|}{$\begin{array}{l}\text { Fig. } 5 \text { Meta-analysis of the mRNA expression levels of NEK2, NDC8C } \\
\text { and CEP } 250 \text { in five HCC vs. normal liver studies. The rank of a gene } \\
\text { is the median rank for that gene across each of the analyses. The } p \\
\text { value for a gene is its } p \text { value for the median-ranked analysis. Red } \\
\text { represents high mRNA expression level, and blue represents low } \\
\text { mRNA expression level. The darker the color, the more obvious the } \\
\text { difference. The five studies were Chen Liver (1), Mas Liver (2), Roessler } \\
\text { Liver (3), Roessler Liver 2 (4), and Wurmbach Liver (5) }\end{array}$} \\
\hline
\end{tabular}

determine whether there were any relationship between the genetic alterations of NEK2, NDC80 and CEP250. The results showed that the relationships between the alterations of the three genes were all co-occurrence (Table 5). The correlation coefficient between NEK2 and NDC80 or CEP250 mRNA expression were 0.87 and 0.59 , respectively (Additional file 5: Figure S5). The overall survival and disease/progression-free survival rates for HCC patients with NEK2, NDC80 and CEP250 genetic alterations were significantly lower than those without the alterations of the three gene $(p=0.0426$ and 0.0341 , respectively, Fig. 7).

\section{Discussion}

Identification of the differences and mechanisms of genes involved in HCC are of great significance for biomarkers and therapeutic targets. NEK2 is a microtubule-binding protein that regulates spindles in human cells. Overexpression of NEK2 mRNA can lead to chromosomal instability, abnormal cell proliferation and drug resistance [13]. Studies showed that NEK2 mRNA 


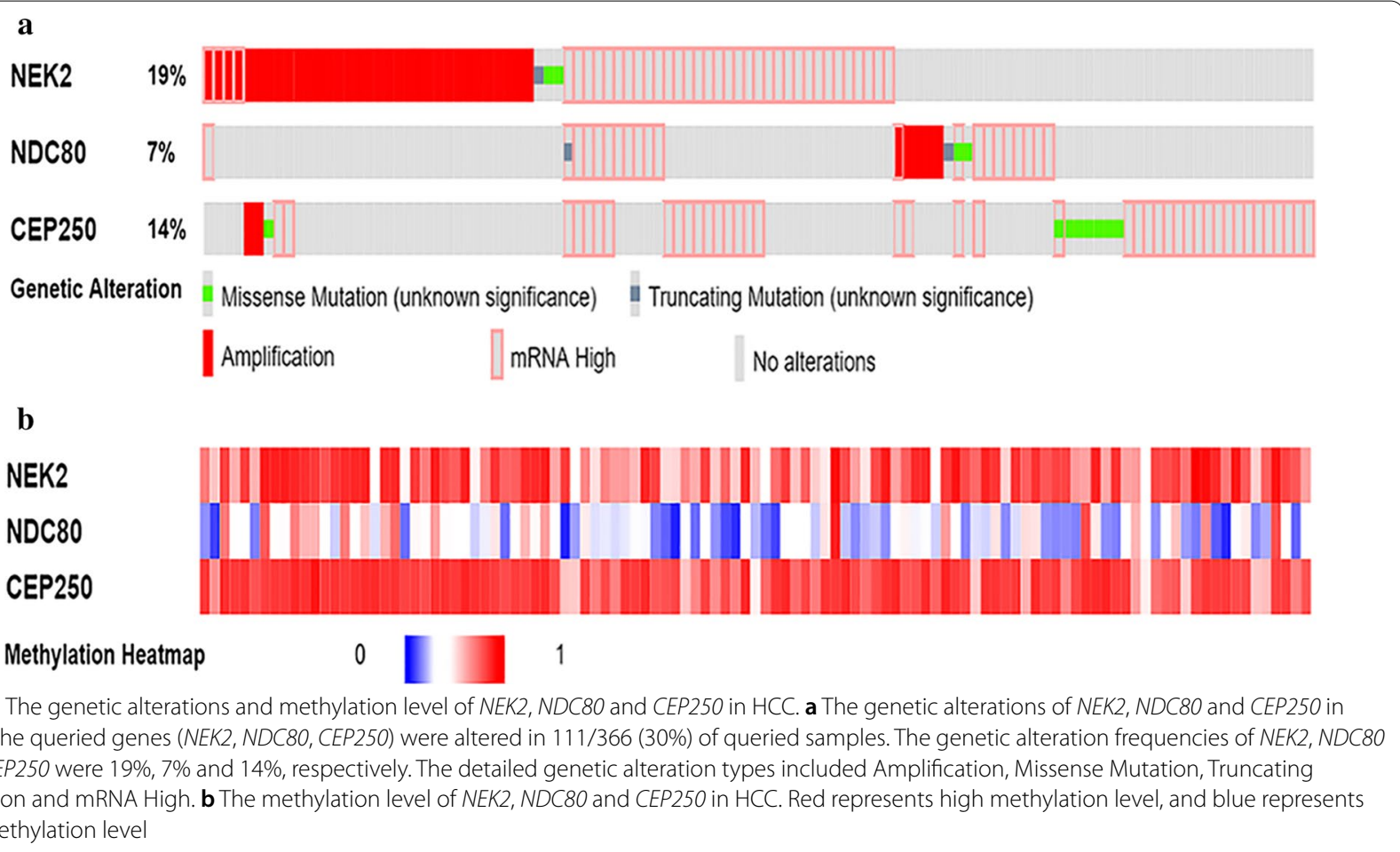

Table 5 Relationships between the genetic alterations of NEK2, NDC80 and CEP250

\begin{tabular}{llllll}
\hline Gene $\mathbf{A}$ & Gene $\mathbf{B}$ & Log odds ratio & $\boldsymbol{p}$ value & Adjusted $\boldsymbol{p}$ value & Tendency \\
\hline NEK2 & CEP250 & 1.267 & $<0.001$ & $<0.001$ & Co-occurrence \\
NEK2 & NDC80 & 1.182 & 0.006 & 0.017 & Co-occurrence \\
NDC80 & CEP250 & 1.27 & 0.006 & 0.018 & Co-occurrence \\
\hline
\end{tabular}

expression was abnormal in HCC. A study in 63 cases of $\mathrm{HCC}$ and matched adjacent non-tumorous liver tissues showed that the mRNA expression of NEK2 was increased, and compared to patients with low mRNA expression, patients with high NEK2 mRNA expression had a poor prognosis. Possible mechanism was that NEK2 influenced the invasion and metastasis of HCC by activating AKT signaling pathway and promoting the expression of matrix metalloproteinases-2 (MMP2) protein [37]. Another study also demonstrated that the prognosis of HCC patients with high mRNA expression of NEK2 were poorer than patients with low expression [38]. However, study has shown that the mRNA expression of NEK2 in HCC tissues was lower than adjacent normal tissues, and was associated with larger tumor diameter, higher alpha-fetoprotein (AFP) concentration, higher tumor stage, worse prognosis and shorter survival time [39]. The differences between the studies need to be clarified by more studies.
In the present study, the mRNA expression of NEK2 in HCC was systematically examined by multiple databases. Results showed that NEK2 mRNA was highly expressed in $\mathrm{HCC}$, and $\mathrm{HCC}$ patients with high NEK2 mRNA expression had poor prognosis. MAD1L1, CEP250, MAPK1, NDC80, PPP1CA, PPP1R2 and NEK11 were found to be the possible upstream or downstream interacting proteins of NEK2. Among these proteins, MAD1L1 was the upstream protein and CEP250, MAPK1, NDC80, NEK11, PPP1CA and PPP1R2 were the downstream proteins of NEK2. NEK11 was both the upstream and downstream protein of NEK2. PPI network confirmed the relationship between NEK2 and its interacting proteins, and NDC80 and CEP250 were found to be NEK2 key interacting proteins. STRING database displayed some GO terms and KEGG pathways that NEK2 and its interacting proteins participated in. Functional pathways by DAVID and KOBAS 3.0 databases also showed that NEK2 and its interacting proteins were 

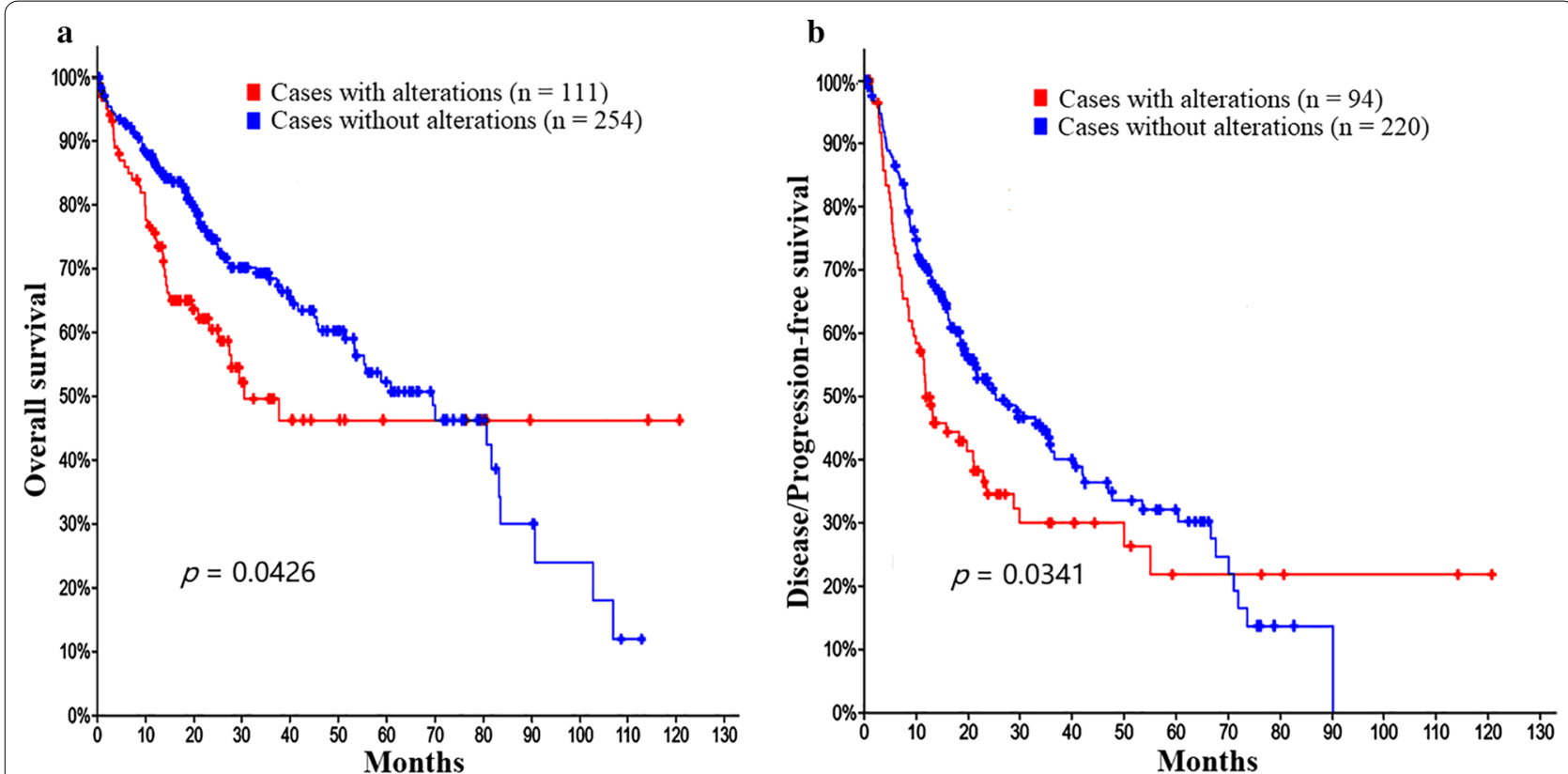

Fig. 7 Effects of NEK2, NDC80 and CEP250 genetic alterations on overall survival (a) and disease/progression-free survival (b) of HCC patients by cBioPortal database analyses

involved in important biological processes. Of note, the analysis results of the databases had some differences. To increase the reliability of the results, Reactome database was chosen for further verification and the results showed that NEK2, NDC80, CEP250, MAPK1 and PPP1CA all participated in important biological pathways. Cell cycle was consistently presented in all the three pathway analyzing tools and mitotic prometaphase, the subset of cell cycle, was the most significant pathway which NEK2 and its interacting proteins involved in. Further analysis indicated that mRNA expressions of the three genes (CCNB1, CCNB2 and CDK1) involved in mitotic prometaphase all had high correlation coefficient with NEK2 mRNA expression. We infer that there may be some interrelations between NEK2 and CCNB1, CCNB2 or CDK1, indirectly suggesting the role of NEK2 in mitotic prometaphase pathway. Kaplan-Meier survival analyses of NEK2 and its interacting proteins in HCC data downloaded from TCGA database showed that only NEK2, NDC80 and CEP250 had significant influence on the prognosis of HCC patients. Taken together, NEK2, NDC80 and CEP250 might play important roles in the pathogenesis of HCC.

Oncomine database analyses showed that NEK2, NDC80 and CEP250 mRNA were over-expression in HCC. cBioPortal database analyses indicated that methylations, genetic and epigenetic alterations of $N E K 2$, NDC80 and CEP250 were found in HCC samples. The survival time of HCC patients with NEK2, NDC80 and
CEP250 genetic alterations were significantly shorter than those without. In univariate Cox regression analyses, NEK2, NDC80 and CEP250 mRNA expression were all significantly related to HCC patients' survival. In multivariate Cox regression model, only NDC80 was the independent predictor of HCC prognosis. These outcomes indicated the critical role of NDC80 in HCC. cBioPortal database revealed the alterations of the three genes were co-occurrence, and clinical correlation analysis also showed the mRNA expression of NDC80, CEP250 and NEK2 were positively correlated. Genensense demonstrated that NDC80 and CEP250 were the downstream protein of NEK2. Therefore, NEK2 may play its role in HCC through interaction with NDC80 and CEP250, especially NDC80. Of course, studies are required to confirm this hypothesis.

NDC80 acts as a component of the essential kinetochore-associated NDC80 complex, plays a role in chromosome congression and is essential for the end-on attachment of the kinetochores to spindle microtubules. NDC80 mRNA expression levels in HCC tissues were found to be significantly higher than those in the adjacent tissues, and NDC80 was believed to contribute to HCC progression by reducing apoptosis and overcoming cell cycle arrest [40]. NDC80 silencing was shown to significantly reduce Hep3 $\mathrm{B}$ cell proliferation and colony formation [41]. CEP250 encodes a core centrosomal protein contributed to the centrosome cohesion, centriole biogenesis, and centrosome duplication processes at 
different cell cycle stages. Phosphorylation of the C-terminal region of CEP250 by NEK2A is essential for promoting centrosome duplication. The phosphorylation of CEP250 induces the release of rootletin from the distal end of the centriole, which in turn leads to the centrosome disjunction [42]. Studies have confirmed that NDC80 and CEP250, in connection with NEK2, participated in the mechanism of some cancers. For example, NEK2 regulated G2/M phases through phosphorylation of HEC1 [20], and HEC1 was crucial for faithful chromosome segregation and high-expression in most cancer cells. HEC1 is a homologous to NDC80 protein located at the budding yeast kinetochore during mitosis [20]. C-Nap1 (also known as CEP250) was demonstrated to be very important at the onset of mitosis and have an dynamic association with NEK2 [43]. It is suggested that NEK2 can phosphorylate multiple sites of C-Nap1 on the C-terminal domain (CTD), rather than a specific site, changing the overall charge of this domain and perturbing oligomerization and centrosome localization. These studies showed the strong connection between NDC80, CEP250 and NEK2.

MAD1L1, a component of the mitotic spindle-assembly checkpoint, belongs to MAD (mitotic arrest deficiency) family. MAD1L1 may play a pathogenic role in various types of human cancer [44] including HCC [45]. MAD1 interacts with NEK2A via a leucine zippercontaining domain located at the $\mathrm{C}$ terminus of $M A D 1$ in vitro and in vivo [17]. MAPK1 encodes a member of the MAP kinase family. MAP kinases, also known as extracellular signal-regulated kinases (ERKs), act as an integration point for multiple biochemical signals, and are involved in a wide variety of cellular processes such as proliferation, differentiation, transcription regulation and development. Studies have demonstrated that MAPK signaling pathway plays an important role in HCC $[46,47]$. NEK2 may regulate proliferation, apoptosis, and other biological behaviors of HCC via MAPK signal pathway [48]. PPP1CA encodes protein phosphatase 1 (PP1) that is essential for cell division and participates in the regulation of glycogen metabolism, muscle contractility and protein synthesis. Few studies have presented its role in HCC currently. However, PP1 alpha was identified as a likely physiological antagonist of NEK2 [49]. Therefore, PP1 encoded by PPP1CA may influence the function of NEK2. The results from DAVID and KOBAS indicated that MAPK1 and PPP1CA were enriched in most pathways whereas NEK2 did not enriched in most pathways. This may be related to the fact that data about the roles of NEK2 in these pathways are still rare at present. STRING database verified that there was weak interaction between MAPK1, PPP1CA and NEK2. NEK2 might play its roles through interacting with MAPK1 and
PPP1CA and result in alterations in biological pathways although further studies are needed to clarify this possibility. PPP1R2 belongs to PPP1R (protein phosphatase 1 regulatory) family, which participates in the inhibition of protein serine/threonine phosphatase. Evidence pertaining the role of PPP1R2 in HCC is scarce, but PPP1R42 (protein phosphatase 1, regulatory subunit 42) depletion may reduce the activity of $\mathrm{PP} 1$, leading to activation of NEK2 [50]. Therefore, PPP1R family may assist NEK2 in its role in HCC. NEK11 is a member of the never in mitosis gene A family of kinases, appears to play roles in DNA replication and response to genotoxic stress, and may function with NEK2A in the S-phase checkpoint [22]. Its role in HCC has not been clarified. To sum up, MAD1L1, MAPK1, PPP1CA, PPP1R2, and NEK11 all have something to do with NEK2. Certainly, the detailed roles of these proteins in HCC need to be verified.

In this study, there were some contradictory results. For example, the molecular function of GO terms in STRING database was enriched by the interesting proteins, but the DAVID database did not show similar result. The number of enriched proteins in the pathways of STRING database was higher than in those of DAVID and KOBAS 3.0 databases. Also, MAPK1 and PPP1CA were enriched in most KEGG pathways, but the interesting proteins (NEK2, NDC80 and CEP250) did not enriched in KEGG pathways. The inconsistencies of pathways among different analyses in our study might be related to the differences of data sources and analyzing focus in different databases. STRING and Reactome databases contain more data from different sources than DAVID and KOBAS 3.0 databases. Reactome database focuses on combining signaling, metabolic molecules and biological pathways into a vivid map. The core unit of the Reactome data model is the reaction. The functions of DAVID and KOBAS 3.0 databases concentrate on annotation and functional gene set enrichment. Differences between databases may lead to different results. However, there was a consistency between the different databases. Namely, cell cycle pathway was presented in all the analyses. Therefore, NEK2 and its interacting proteins might be implicated in the pathogenesis of HCC through cell cycle pathway, especially mitotic prometaphase pathway, a subset of cell cycle. NEK2, NDC80, CEP250 and MAD1L1 were the proteins enriched in mitotic prometaphase pathway. These results support that NEK2, NDC80 and CEP250 may coordinately play important roles in influencing HCC development. In univariate Cox regression model, NEK2, NDC80, and CEP250 were all significantly associated with HCC patient's survival. In multivariate Cox regression model, NDC80 was an independent predictor for 
HCC patients' survival, but NEK2 and CEP250 were not independent predictors. We speculate that the function of NEK2 was influenced by NDC80. In univariate Cox regression model, the interrelationship exited between NEK2, NDC80 and CEP250, suggesting the influential role of NEK2 and CEP250 on the survival of HCC patients. In multivariate Cox regression model, the interrelationship between NEK2, NDC80 and CEP250 was diminished, and the influence of NEK2 and CEP250 on HCC patients' survival disappeared. We speculate that NEK2 alone may play a weak role in HCC. Genesense analysis showed that NDC80 was the downstream protein of NEK2. Therefore, NEK2 might exert its effect on HCC mainly by influencing NDC80. CCNB1, CCNB2 and CDK1 were all proteins that participate in mitotic prometaphase pathway, and the mRNA expression of the three genes have been shown to have strong positive correlation with NEK2 mRNA expression. Genesense website did not show the upstream and downstream relationship between CCNB1, CCNB2, CDK1 and NEK2, but the high correlation coefficient suggested that there must be some relationship between these proteins. NEK2, NDC80, and CEP250 may play some roles together with CCNB1, CCNB2 and CDK1 in mitotic prometaphase pathway. The survival analysis outcome in our study was similar with the study by Li et al. [13]. The clinical covariates in the study of Li et al. could not be incorporated in our analysis because our study used the median value of NEK2 mRNA expression while Li et al. used the cut-off point of NEK2 mRNA expression defined by the receiver operating characteristic curve to categorize the HCC patients to high or low expression groups. However, the conclusions of the two studies reach a consensus, that is, the prognosis of HCC patients with high NEK2 mRNA expression was poorer than those with low expression.

Small molecule therapies targeting NEK2 and NDC80 in different tumors have been reported. NEK2 siRNA was found to inhibit tumor growth in a subcutaneous xenograft mouse model of pancreatic cancer, prolong the survival time in an intraperitoneal xenograft mouse model and efficiently prevent the progression of liver metastasis [51]. Combination of NEK2 siRNA and chemotherapeutic agents may be effective treatment for colorectal cancer [52]. NDC80 might be a good targeting option in suppressing breast cancer tumor growth, and dual targeting of NEK 2 and NDC80 might improve the prognosis [53]. Currently, there have been no studies on molecular therapy by targeting CEP250. Whether targeting NEK2, NDC80 or CEP250 alone or in various combinations may be therapeutically feasible for HCC still needs experimental studies in future.

\section{Conclusions}

In summary, the systematic bioinformatics analyses in this study showed that NEK2 and its interacting proteins (MAD1L1, CEP250, MAPK1, NDC80, PPP1CA, PPP1R2, NEK11), especially NEK2 key interacting proteins (NDC80 and CEP250), are significantly involved in HCC. Mitotic prometaphase pathway, a subset of cell cycle pathway, might be the crucial pathway that NEK2, NDC80 and CEP250 are involved in the pathogenesis of HCC. There are strong interactions between NEK2, NDC80 and CEP250. The mRNA expressions of NEK2, NDC80 and CEP250 were significantly associated with HCC patients' survival. Patients with high mRNA expression and genetic alterations of NEK2, NDC80 and $C E P 250$ had poor prognosis. These findings provide evidence for the potential application of NEK2 as well as NDC80 and CEP250 as biomarkers for HCC diagnosis and HCC patient prognosis and as targets for HCC therapy.

\section{Supplementary information}

Supplementary information accompanies this paper at https://doi. org/10.1186/s12920-020-00812-y.

Additional file 1. Figure S1: Flow chart of the study and the sample numbers for each data mining part.

Additional file 2. Figure S2: The pathways that NEK2 and its interacting proteins participate in by Reactome database analysis. The red lines in the figure fireworks present the pathways that NEK2 and its interacting proteins may be involved in, and the blue lines present the pathways that NEK2 and its interacting proteins may not be involved in.

Additional file 3. Figure S3: The details of mitotic prometaphase pathway. The purple boxes represent the main biological processes that NEK2 and its interacting proteins may involve in.

Additional file 4. Figure S4: The correlation of mRNA expression between NDC80, CEP250, CCNB1, CCNB2, CDK1 and NEK2 from TCGA database. A. NEK2 vs. NDC80; B. NEK2 vs. CEP250; C. NEK2 vs. CCNB1; D NEK2 vs. CCNB2; E. NEK2 vs. CDK1.

Additional file 5. Figure S5: The correlation of mRNA expression from cBioPortal database. A. NEK2 vs. NDC80; B. NEK2 vs. CEP250.

Additional file 6. Table S1: The characteristics of 373 HCC patients from TCGA database.

\section{Abbreviations}

AFP: Alpha-fetoprotein; BP: Biological process; CC: Cellular component; CEP250: Centrosomal protein 250 kDa; CNA: Copy number alteration; CTD: C-terminal domain; DAVID: The Database for Annotation, Visualization and Integrated Discovery; ERK: Extracellular signal-regulated kinase; FDR: False discovery rate; GISTIC: Genomic identification of significant targets in cancer; GO: Gene Ontology; HCC: Hepatocellular carcinoma; KEGG: Kyoto Encyclopedia of Genes and Genomes; KOBAS: KEGG Orthology Based Annotation System; MAD: Mitotic arrest deficiency; MAD1L1: MAD1 mitotic arrest deficient-like 1 (yeast); MAPK1: Mitogen-activated protein kinase 1; MF: Molecular function; MMP-2: Matrix metalloproteinases-2; NDC80: Nuclear division cycle 80; NEK2: Never in mitosis gene A-related kinase 2; NEK11: NIMA (never in mitosis gene a)-related kinase 11; NuMA: Nuclear mitotic apparatus protein; PP1: Protein phosphatase 1; PPI: Protein-protein interaction; PPP1CA: Protein phosphatase 1 catalytic subunit alpha; PPP1R: Protein phosphatase 1 regulatory; PPP1R2: Protein phosphatase 1, regulatory (inhibitor) subunit 2; PPP1R42: Protein phosphatase 1, regulatory subunit 42; SD: Standard deviation; STRING: Search 
Tool for the Retrieval of Interacting Genes/Proteins; TCGA: The Cancer Genome Atlas; UALCAN: University of Alabama Cancer Database.

\section{Acknowledgements}

Not Applicable.

\section{Authors' contributions}

$L Z, X F$ and $Z L$ conceived, designed, and managed the study. XW, HD, XZ, KZ, and SH involved in the collection and analysis of the data. LZ and ZL wrote the manuscript. NL and QH involved in the data analysis. ZL obtained the funding. All authors participated in the revision of the manuscript and approved the submission. All authors read and approved the final manuscript.

\section{Funding}

This study was supported in part by the National Natural Science Foundation of China (Grant no. 81371798). The funder has no role in the study design, data collection and analysis, decision to publish, or preparation of the manuscript.

\section{Availability of data and materials}

The datasets used and analyzed in this study are available from the corresponding websites of the databases, including UALCAN: https://ualca n.path.uab.edu/cgi-bin/TCGAExResultNew2.pl?genenam $=$ NEK2\&ctype $=\mathrm{LIHC}$; GeneSense: https://www.biomedsense.org/g_interloop2.php?g=NEK2; STRING: https://string-db.org/cgi/network.pl?taskld=sYn3BXkN9fGj; DAVID: https://david-d.ncifcrf.gov/ (The analysis criteria in the method section of our article need to be re-entered to see our results); KOBAS3.0: https:// kobas.cbi.pku.edu.cn/kobas3/genelist/ (The analysis criteria in the method section of our article need to be re-entered to see our results); Reactome: https://reactome.org/PathwayBrowser/\#/DTAB=AN\&ANALYSIS=MjAyM DA2MTgwMDAONTIfNjc5\&FILTER=resource:UNIPROT; Oncomine: https:// www.oncomine.org/resource/login.html (Registration is needed to login into the database) and cBioPortal: https://www.cbioportal.org/results/oncop rint?Action=Submit\&RPPA_SCORE_THRESHOLD $=2.0 \& Z$ SCORE_THRES HOLD $=2.0 \&$ cancer_study_list=lihc_tcga\&case_set_id=lihc_tcga_cnase q\&data_priority $=0 \&$ gene $\_$list $=$NEK2\%252C\%2520NDC $80 \% 252$ C $\% 2520 \mathrm{C}$ EP250\&geneset_list=\%20\&genetic_profile_ids_PROFILE_COPY_NUMBE R_ALTERATION=lihc_tcga_gistic\&genetic_profile_ids_PROFILE_MUTAT ION_EXTENDED=lihc_tcga_mutations\&profileFilter $=0$ \&tab_index=tab_visua lize\&genetic_profile_ids_PROFILE_MRNA_EXPRESSION=lihc_tcga_rna_seq_ V2_mrna_median_Zscores. The results were often aggregated from multiple databases and analyzed online without the need to download the data. The data of the 373 HCC patients from TCGA database were downloaded from https://portal.gdc.cancer.gov/repository?cases_offset=200\&facet Tab=cases\&filters=\%7B\%22op\%22\%3A\%22and\%22\%2C\%22content $\% 22 \%$ 3A\%5B\%7B\%22op\%22\%3A\%22in\%22\%2C\%22content\%22\%3A\%7B\%22fie Id\%22\%3A\%22cases.primary_site\%22\%2C\%22value\%22\%3A\%5B\%22liver\%20 and\%20intrahepatic\%20bile\%20ducts\%22\%5D\%7D\%7D\%2C\%7B\%22op\% 22\%3A\%22in\%22\%2C\%22content $\% 22 \% 3$ A\%7B\%22field\%22\%3A\%22cas es.project.project_id\%22\%2C\%22value\%22\%3A\%5B\%22TCG A-LIHC\%22\%5D\%7D\%7D\%5D\%7D\&searchTableTab=cases. All data generated during this study are included in this published article.

\section{Ethics approval and consent to participate}

Not applicable.

\section{Consent for publication}

Not applicable.

\section{Competing interests}

The authors declare that they have no competing interests.

\section{Author details}

${ }^{1}$ Department of Infectious Diseases, First Affiliated Hospital of Xi'an Jiaotong University, No. 277 Yanta West Road, Xi'an 710061, Shaanxi Province, People's Republic of China. ${ }^{2}$ Xi'an Medical University, Xi'an 710021, Shaanxi Province, People's Republic of China.

Received: 26 April 2019 Accepted: 25 June 2020

Published online: 27 October 2020

\section{References}

1. Bertuccio P, Turati F, Carioli G, Rodriguez T, La Vecchia C, Malvezzi M, et al. Global trends and predictions in hepatocellular carcinoma mortality. J Hepatol. 2017;67(2):302-9.

2. Goldberg DS, Taddei TH, Serper M, Mehta R, Dieperink E, Aytaman A, et al. Identifying barriers to hepatocellular carcinoma surveillance in a national sample of patients with cirrhosis. Hepatology. 2017;65(3):864-74.

3. Wu W, Baxter JE, Wattam SL, Hayward DG, Fardilha M, Knebel A, et al. Alternative splicing controls nuclear translocation of the cell cycle-regulated Nek2 kinase. J Biol Chem. 2007;282(36):26431-40.

4. Fry AM. The Nek2 protein kinase: a novel regulator of centrosome structure. Oncogene. 2002;21(40):6184-94.

5. Conroy PC, Saladino C, Dantas TJ, Lalor P, Dockery P, Morrison CG. C-NAP1 and rootletin restrain DNA damage-induced centriole splitting and facilitate ciliogenesis. Cell Cycle. 2012;11(20):3769-78.

6. Wen S, Liu Y, Yang M, Yang K, Huang J, Feng D. Increased NEK2 in hepatocellular carcinoma promotes cancer progression and drug resistance by promoting PP1/Akt and Wnt activation. Oncol Rep. 2016;36(4):2193-9.

7. Zhou W, Yang Y, Xia J, Wang H, Salama ME, Xiong W, et al. NEK2 induces drug resistance mainly through activation of efflux drug pumps and is associated with poor prognosis in myeloma and other cancers. Cancer Cell. 2013;23(1):48-62.

8. Ning Z, Wang A, Liang J, Liu J, Zhou T, Yan Q, et al. Abnormal expression of Nek2 in pancreatic ductal adenocarcinoma: a novel marker for prognosis. Int J Clin Exp Pathol. 2014;7(5):2462-9.

9. Liu X, Gao Y, Lu Y, Zhang J, Li L, Yin F. Upregulation of NEK2 is associated with drug resistance in ovarian cancer. Oncol Rep. 2014;31(2):745-54.

10. Neal CP, Fry AM, Moreman C, McGregor A, Garcea G, Berry DP, et al. Overexpression of the Nek2 kinase in colorectal cancer correlates with beta-catenin relocalization and shortened cancer-specific survival. J Surg Oncol. 2014;110(7):828-38.

11. Marina M, Saavedra HI. Nek2 and Plk4: prognostic markers, drivers of breast tumorigenesis and drug resistance. Front Biosci (Landmark Ed). 2014;19:352-65.

12. Lai $X B, N i e Y Q$, Huang $H L$, Li YF, Cao CY, Yang H, et al. NIMA-related kinase 2 regulates hepatocellular carcinoma cell growth and proliferation. Oncol Lett. 2017;13(3):1587-94.

13. Li G, Zhong Y, Shen Q, Zhou Y, Deng X, Li C, et al. NEK2 serves as a prognostic biomarker for hepatocellular carcinoma. Int $J$ Oncol. 2017:50(2):405-13.

14. Therneau TM, Grambsch PM. Estimating the survival and hazard functions. In: Therneau TM, Grambsch PM, editors. Modeling survival data: extending the cox model. New York: Springer; 2000. p. 7-37.

15. Chandrashekar DS, Bashel B, Balasubramanya SAH, Creighton CJ, Ponce-Rodriguez I, Chakravarthi B, et al. UALCAN: a portal for facilitating tumor subgroup gene expression and survival analyses. Neoplasia. 2017;19(8):649-58.

16. Chen Z, Zhang T, Lin J, Yan Z, Wang Y, Zheng W, et al. GeneSense: a new approach for human gene annotation integrated with protein-protein interaction networks. Sci Rep. 2014;4:4474.

17. Lou Y, Yao J, Zereshki A, Dou Z, Ahmed K, Wang H, et al. NEK2A interacts with MAD1 and possibly functions as a novel integrator of the spindle checkpoint signaling. J Biol Chem. 2004;279(19):20049-57.

18. Helps NR, Luo X, Barker HM, Cohen PT. NIMA-related kinase 2 (Nek2), a cell-cycle-regulated protein kinase localized to centrosomes, is complexed to protein phosphatase 1. Biochem J. 2000;349(Pt 2):509-18.

19. Lou Y, Xie W, Zhang DF, Yao JH, Luo ZF, Wang YZ, et al. Nek2A specifies the centrosomal localization of Erk2. Biochem Biophys Res Commun. 2004;321(2):495-501.

20. Chen Y, Riley DJ, Zheng L, Chen PL, Lee WH. Phosphorylation of the mitotic regulator protein $\mathrm{Hec} 1$ by Nek2 kinase is essential for faithful chromosome segregation. J Biol Chem. 2002;277(51):49408-16.

21. Eto M, Elliott E, Prickett TD, Brautigan DL. Inhibitor-2 regulates protein phosphatase-1 complexed with NimA-related kinase to induce centrosome separation. J Biol Chem. 2002;277(46):44013-20.

22. Noguchi K, Fukazawa H, Murakami Y, Uehara Y. Nucleolar Nek11 is a novel target of Nek2A in G1/S-arrested cells. J Biol Chem. 2004;279(31):32716-27.

23. Szklarczyk D, Franceschini A, Wyder S, Forslund K, Heller D, Huerta-Cepas J, et al. STRING v10: protein-protein interaction networks, integrated over the tree of life. Nucleic Acids Res. 2015; 43(Database issue):D447-52. 
24. Vinayagam A, Stelzl U, Foulle R, Plassmann S, Zenkner M, Timm J, et al. A directed protein interaction network for investigating intracellular signal transduction. Sci Signal. 2011;4(189):rs8.

25. Fry AM, Mayor T, Meraldi P, Stierhof YD, Tanaka K, Nigg EA. C-Nap1, a novel centrosomal coiled-coil protein and candidate substrate of the cell cycleregulated protein kinase Nek2. J Cell Biol. 1998;141(7):1563-74.

26. Fogeron $\mathrm{ML}$, Muller $\mathrm{H}$, Schade $\mathrm{S}$, Dreher F, Lehmann $\mathrm{V}$, Kuhnel $\mathrm{A}$, et al. LGALS3BP regulates centriole biogenesis and centrosome hypertrophy in cancer cells. Nat Commun. 2013:4:1531.

27. da Huang W, Sherman BT, Lempicki RA. Systematic and integrative analysis of large gene lists using DAVID bioinformatics resources. Nat Protoc. 2009;4(1):44-57.

28. Ai C, Kong L. CGPS: A machine learning-based approach integrating multiple gene set analysis tools for better prioritization of biologically relevant pathways. J Genet Genom. 2018;45(9):489-504.

29. Fabregat A, Jupe S, Matthews L, Sidiropoulos K, Gillespie M, Garapati $P$, et al. The reactome pathway knowledgebase. Nucleic Acids Res. 2018;46(D1):D649-55.

30. Grossman RL, Heath AP, Ferretti V, Varmus HE, Lowy DR, Kibbe WA, et al. Toward a shared vision for cancer genomic data. N Engl J Med. 2016;375(12):1109-12.

31. Cohen-Eliav M, Golan-Gerstl R, Siegfried Z, Andersen CL, Thorsen K, Orntoft TF, et al. The splicing factor SRSF6 is amplified and is an oncoprotein in lung and colon cancers. J Pathol. 2013;229(4):630-9.

32. Chen X, Cheung ST, So S, Fan ST, Barry C, Higgins J, et al. Gene expression patterns in human liver cancers. Mol Biol Cell. 2002;13(6):1929-39.

33. Mas VR, Maluf DG, Archer KJ, Yanek K, Kong X, Kulik L, et al. Genes involved in viral carcinogenesis and tumor initiation in hepatitis $C$ virusinduced hepatocellular carcinoma. Mol Med. 2009;15(3-4):85-94.

34. Roessler S, Jia HL, Budhu A, Forgues M, Ye QH, Lee JS, et al. A unique metastasis gene signature enables prediction of tumor relapse in early-stage hepatocellular carcinoma patients. Cancer Res. 2010;70(24):10202-12.

35. Wurmbach E, Chen YB, Khitrov G, Zhang W, Roayaie S, Schwartz M, et al. Genome-wide molecular profiles of HCV-induced dysplasia and hepatocellular carcinoma. Hepatology. 2007;45(4):938-47.

36. Gao J, Aksoy BA, Dogrusoz U, Dresdner G, Gross B, Sumer SO, et al. Integrative analysis of complex cancer genomics and clinical profiles using the cBioPortal. Sci Signal. 2013; 6(269):pl1.

37. Zhang $Y$, Wang W, Wang $Y$, Huang $X$, Zhang Z, Chen B, et al. NEK2 promotes hepatocellular carcinoma migration and invasion through modulation of the epithelial-mesenchymal transition. Oncol Rep. 2018;39(3):1023-33.

38. Fu L, Liu S, Wang H, Ma Y, Li L, He X, et al. Low expression of NEK2 is associated with hepatocellular carcinoma progression and poor prognosis. Cancer Biomark. 2017;20(1):101-6.

39. Chen L, Guo P, He Y, Chen Z, Chen L, Luo Y, et al. HCC-derived exosomes elicit HCC progression and recurrence by epithelial-mesenchymal transition through MAPK/ERK signalling pathway. Cell Death Dis. 2018;9(5):513.
40. Ju LL, Chen L, Li JH, Wang YF, Lu RJ, Bian ZL, et al. Effect of NDC80 in human hepatocellular carcinoma. World J Gastroenterol. 2017;23(20):3675-83.

41. Liu B, Yao Z, Hu K, Huang H, Xu S, Wang Q, et al. ShRNA-mediated silencing of the $\mathrm{Ndc} 80$ gene suppress cell proliferation and affected hepatitis B virus-related hepatocellular carcinoma. Clin Res Hepatol Gastroenterol. 2016;40(3):297-303.

42. Kumar A, Rajendran V, Sethumadhavan R, Purohit R. CEP proteins: the knights of centrosome dynasty. Protoplasma. 2013;250(5):965-83.

43. Hardy T, Lee M, Hames RS, Prosser SL, Cheary DM, Samant MD, et al. Multisite phosphorylation of C-Nap1 releases it from Cep135 to trigger centrosome disjunction. J Cell Sci. 2014;127(Pt 11):2493-506.

44. Tsukasaki K, Miller CW, Greenspun E, Eshaghian S, Kawabata H, Fujimoto $\mathrm{T}$, et al. Mutations in the mitotic check point gene, MAD1L1, in human cancers. Oncogene. 2001;20(25):3301-5.

45. Sze KM, Ching YP, Jin DY, Ng IO. Role of a novel splice variant of mitotic arrest deficient 1 (MAD1), MAD1 beta, in mitotic checkpoint control in liver cancer. Cancer Res. 2008;68(22):9194-201.

46. Zhao F, Gong X, Liu A, Lv X, Hu B, Zhang H. Downregulation of Nedd4L predicts poor prognosis, promotes tumor growth and inhibits MAPK/ERK signal pathway in hepatocellular carcinoma. Biochem Biophys Res Commun. 2018;495(1):1136-43.

47. Wang J, Sun P, Chen Y, Yao H, Wang S. Novel 2-phenyloxypyrimidine derivative induces apoptosis and autophagy via inhibiting PI3K pathway and activating MAPK/ERK signaling in hepatocellular carcinoma cells. Sci Rep. 2018;8(1):10923.

48. Zhang MX, Xu XM, Zhang P, Han NN, Deng JJ, Yu TT, et al. Effect of silencing NEK2 on biological behaviors of HepG2 in human hepatoma cells and MAPK signal pathway. Tumour Biol. 2016;37(2):2023-35.

49. Meraldi P, Nigg EA. Centrosome cohesion is regulated by a balance of kinase and phosphatase activities. J Cell Sci. 2001;114(Pt 20):3749-57.

50. DeVaul N, Wang R, Sperry AO. PPP1R42, a PP1 binding protein, regulates centrosome dynamics in ARPE-19 cells. Biol Cell. 2013;105(8):359-71.

51. Kokuryo T, Hibino S, Suzuki K, Watanabe K, Yokoyama Y, Nagino M, et al. Nek2 siRNA therapy using a portal venous port-catheter system for liver metastasis in pancreatic cancer. Cancer Sci. 2016;107(9):1315-20.

52. Suzuki K, Kokuryo T, Senga T, Yokoyama Y, Nagino M, Hamaguchi M. Novel combination treatment for colorectal cancer using Nek2 siRNA and cisplatin. Cancer Sci. 2010;101(5):1163-9.

53. Wu G, Qiu XL, Zhou L, Zhu J, Chamberlin R, Lau J, et al. Small molecule targeting the Hec1/Nek2 mitotic pathway suppresses tumor cell growth in culture and in animal. Cancer Res. 2008;68(20):8393-9.

\section{Publisher's Note}

Springer Nature remains neutral with regard to jurisdictional claims in published maps and institutional affiliations.

Ready to submit your research? Choose BMC and benefit from:

- fast, convenient online submission

- thorough peer review by experienced researchers in your field

- rapid publication on acceptance

- support for research data, including large and complex data types

- gold Open Access which fosters wider collaboration and increased citations

- maximum visibility for your research: over 100M website views per year

At BMC, research is always in progress.

Learn more biomedcentral.com/submissions 